\title{
The Ciona17 Dataset for Semantic Segmentation of Invasive Species in a Marine Aquaculture Environment
}

\author{
Angus Galloway*, Graham W. Taylor*, Aaron Ramsay ${ }^{\dagger}$, Medhat Moussa* \\ * School of Engineering \\ University of Guelph \\ Guelph, ON, Canada \\ \{gallowaa,gwtaylor,mmoussa\}@uoguelph.ca
}

$\dagger$ Department of Agriculture and Fisheries

Government of PEI

Montague, PEI, Canada

\{apramsay@gov.pe.ca\}

\begin{abstract}
An original dataset for semantic segmentation, "Ciona17", is introduced, which to the best of the authors' knowledge, is the first dataset of its kind with pixel-level annotations pertaining to invasive species in a marine environment. Diverse outdoor illumination, a range of object shapes, colour, and severe occlusion provide a significant real world challenge for the computer vision community. An accompanying groundtruthing tool for superpixel labeling, "Truth and Crop", is also introduced. Finally, we provide a baseline using a variant of Fully Convolutional Networks, and report results in terms of the standard mean intersection over union (mIoU) metric.
\end{abstract}

Keywords-semantic segmentation; object segmentation dataset; aquaculture management; aquatic invasive species; Ciona intestinalis; Mytilus edulis

\section{INTRODUCTION}

In long-line mussel farming, the fouling of mussel socks and gear by tunicates, a marine invertebrate species, can impede mussel growth, decrease yield, and reduce overall farm productivity [1]-[4]. Ciona intestinalis are widely considered to be the most problematic of the tunicates in mussel farming due to their rapid proliferation and biomass [1], [3]. Aquaculture industries in The Netherlands, New Zealand, and particularly Prince Edward Island (PEI), Canada, are plagued by Ciona; having the ability to displace other invasive tunicates, such as Styela clava [1], [4].

In response to the Ciona problem, an above-water highpressure treatment system, depicted in Figure 1, has been in use for several years [3], but this design has several significant limitations. Current mitigation systems do remove substantial Ciona biomass, but they also introduce avoidable mussel fall-off, as the force of water jets can exceed the tensile strength of the mussels' byssal threads.

The fall-off problem is further exacerbated during the summer months, when byssal threads are weaker [5], spawning of Ciona is at peak intensity [1], and treatments are most frequent. Furthermore, mussel fall-off is expected to increase with rising ocean acidification [6].
The high-pressure treatment system can be considered open-loop with respect to the extent of infestation on a mussel sock. Quantifying invasive species by surface area, and location, in real-time, is central to the feasibility of an improved closed-loop computer vision-based treatment system. Such a system, paired with electromechanical nozzles, could strategically target invasive species, minimizing mussel fall-off as well as the number of requisite treatments in a growing season.

Measuring the amount of biofouling on mussel socks before and after treatment, is also desirable for making informed management decisions. Given a cleanliness metric, farmers can counteract inconsistent treatments caused by the use of non-standardized equipment (e.g. different nozzles, orientation, water pressure), and varying treatment speeds.

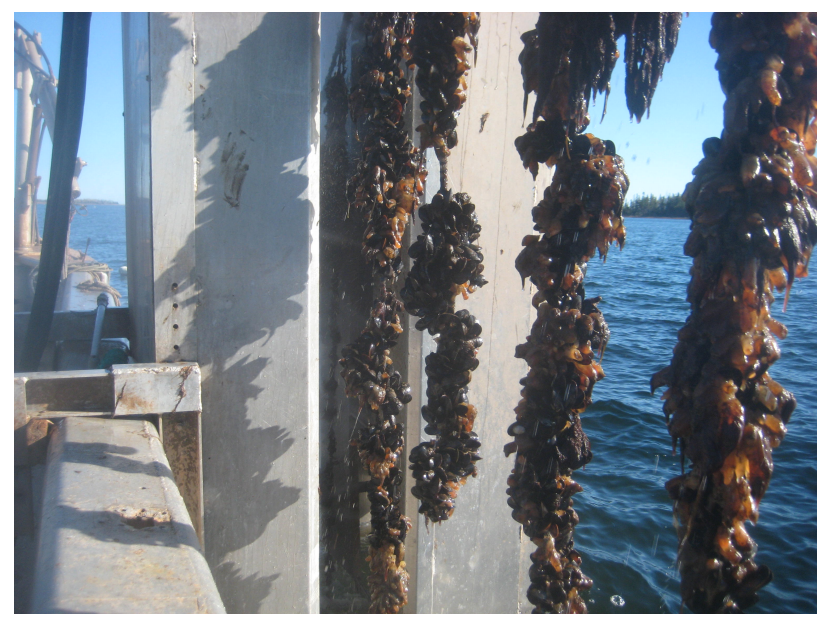

Figure 1. Long-line farmed mussel socks with moderate Ciona infestation (orange gelatinous specimen) about to enter a high-pressure treatment system fixed to hull of vessel. A generic design for this system is available [7], but each farm's equipment is made to order. Best viewed in colour.

The farming context is the motivation for formulating the 
problem as one of semantic segmentation as opposed to simply object detection, since we seek to describe where, what kind, and how much infestation is present. Counting individual object instances is of less importance in mitigation, but may be of scientific interest in other capacities, such as a detailed study of recruitment patterns [2].

\section{RELATED WORK}

In the context of semantic segmentation, to date, several major datasets have served as common benchmarks for assessing algorithm performance. Yet, comparatively few arise from real-world, application specific problems. In this section we discuss some alternative datasets that facilitate supervised semantic segmentation with pixel-level annotations, and draw comparisons with Ciona17.

\section{A. PASCAL Visual Object Classes [8]}

The most recent PASCAL Visual Object Classes (VOC) challenge dataset has 21 object classes (e.g. aeroplane, bicycle, bird), and provides 2,913 pixel-level segmentations [8].

The PASCAL dataset addressed several criticisms of earlier object recognition datasets, which lacked clutter and diverse object orientations. Given that the PASCAL images were drawn pseudo-randomly from the consumer photo sharing website Flickr, they tend to be representative of challenging real-world scenes. Although the PASCAL VOC competitions have ended, the datasets continue to be a standard benchmark, having led to several breakthroughs in the field of deep learning for semantic segmentation [9][11].

\section{B. The Cityscapes Dataset for Semantic Urban Scene Un- derstanding [12]}

This more modern dataset extends PASCAL VOC to highcomplexity urban scenes, and features 5,000 fine pixel-level annotations, and 20,000 self described "coarse" annotations, for 30 different object classes (e.g. person, rider, car, truck) and eight broader categories (e.g. human, vehicle, nature). For testing, only 18 of the sufficiently unique objects are used to assess performance. The dataset organizers employed two forms of quality control regarding the annotations: 1) having 30 images labeled by two different annotators, and 2) coarse labeling of previously fine labeled images. Both measures resulted in greater than $96 \%$ overlap, a testament to high consistency even among numerous annotators.

\section{Comparisons}

Similarly to the Ciona17 dataset, each example in PASCAL consists of an RGB image, and segmentation mask containing integer indices corresponding to an object class. Unlike the PASCAL dataset, Ciona17 does not contain object instance masks, as the practical nature of farming does not require counting of individual species. Furthermore, a single cropped image of modest resolution could potentially contain hundreds of individual Ciona or mussels, making this impractical to label accurately.

In comparing the PASCAL dataset and Ciona17, the latter contains even more occlusion and blending of classes, as the tunicates tend to grow directly on top of, or among, the mussels. The PASCAL dataset does contain some occlusion, such as "person riding a bicycle" or "busy street scene", however many examples feature one or two instances of an unobstructed object, e.g. "aeroplane in the sky". In comparison, many randomly oriented object instances with heavy occlusion, is the norm rather than the exception, in Ciona17.

The Cityscapes dataset is more similar to Ciona17, as they are both application-specific and most examples contain several crowded objects, with some Cityscapes scenes having over a hundred instances. Cityscapes is also highly occluded, with vehicles and pedestrians obstructing views of other people.

\section{The Ciona17 DATASET}

We introduce a ground-truthed dataset derived from highresolution images taken on two different mussel farms ${ }^{1}$ Images were collected with a consumer-grade Canon PowerShot SD1000 7.1MP digital camera mounted on a tripod with ISO fixed to 100. Both farms were located in eastern PEI and experienced significant infestations of Ciona intestinalis. Only one of the farms, "Farm 2", had visible Styela clava.

No specific instruction was given to the farmers regarding the type of crop to show. They were simply told to treat what they had originally intended to do that day. We have high confidence that the data collected is representative of what a typical farm would encounter during a normal treatment cycle. Data collection occurred on two consecutive days near the end of the tunicate spawning season in PEI, in early November 2016. This was the last scheduled day of treatments for Farm 1, as Ciona cease to reproduce below water temperatures of about $8^{\circ} \mathrm{C}[1]$.

\section{A. High Level Overview}

The Ciona17 dataset consists of 1,472 images with corresponding pixel-level segmentations. The images are cropped to a uniform $224 \times 224 \mathrm{px}$, and the following semantic labels were assigned and stored to integer masks:

- 0 - Other

- 1 - Mussel

- 2 - Ciona

- 3 - Styela

- 4 - Void

The "Other" class represented uninteresting background classes that were not explicitly annotated, while "Void" was

\footnotetext{
${ }^{1}$ http://dx.doi.org/10.5683/SP/NTUOK9
} 
used for regions that were difficult to label (e.g. foreground class overlap). The distribution of class labels is shown in Table II. The dataset is split into two training, and one test set. The test set has significantly more Ciona, and facilitates testing how resulting algorithms handle class-imbalance. Both training sets have a similar volume of Ciona, but Training 2 was unique in receiving a void label during annotation, in similar fashion to PASCAL VOC. We are aware of additional complexities in adapting some models to use void labels, such as Fully Convolutional Networks (FCN) [9] implemented in frameworks like TensorFlow [13], therefore we keep Training 2 separate.

Table I

Class BALANCE FOR TRAINING AND TEST DATA FOR FARM 1.

\begin{tabular}{lccccc}
\hline Split & Images & Other $\%$ & Mussel $\%$ & Ciona $\%$ & Void $\%$ \\
\hline Training 1 & 702 & 38 & 49 & 13 & 0 \\
Training 2 & 364 & 35 & 29 & 14 & 19 \\
Test & 334 & 42 & 18 & 40 & 0 \\
\hline
\end{tabular}

\section{B. Truth and Crop Tool}

To facilitate quick ground truthing of full size images, a platform-independent GUI utility "Truth and Crop" was implemented in $\mathrm{Q}^{2}$ This tool let the annotator label whole superpixels, rather than individual pixels, or by drawing polygons. Using the tool, an annotator performs an initial ground truth, and can then click arbitrarily to save cropped image-segmentation mask pairs.

The simple linear iterative clustering (SLIC) superpixel extraction algorithm [14] is used to automatically find boundaries around similar parts or edges in the image. The SLIC algorithm implemented in the Python scikitimage [15] library receives as arguments the number of roughly equal size segments requested, width of Gaussian smoothing kernel, $\sigma$, and a segment compactness factor. Once superpixel clusters have been identified, they can be imposed on the original image, and the annotator assigns a class label to all pixels in a superpixel with a single click. This process is depicted in Figure 2 .

\section{Ground Truthing Procedure}

Images were ground truthed by the primary author after meeting with aquaculture biologists, and spending significant time manually inspecting live mussel socks.

Through the Truth and Crop GUI, SLIC hyperparameters were set manually on a per-image basis, while spending roughly 10-15 minutes per image for the whole annotation procedure. An optional down-sampling factor, usually of no more than two or three, was first applied to the original image in both height and width dimensions. This helped ensure that samples were drawn from a variety of scales, and

\footnotetext{
${ }^{2}$ https://github.com/AngusG/truth-and-crop
}

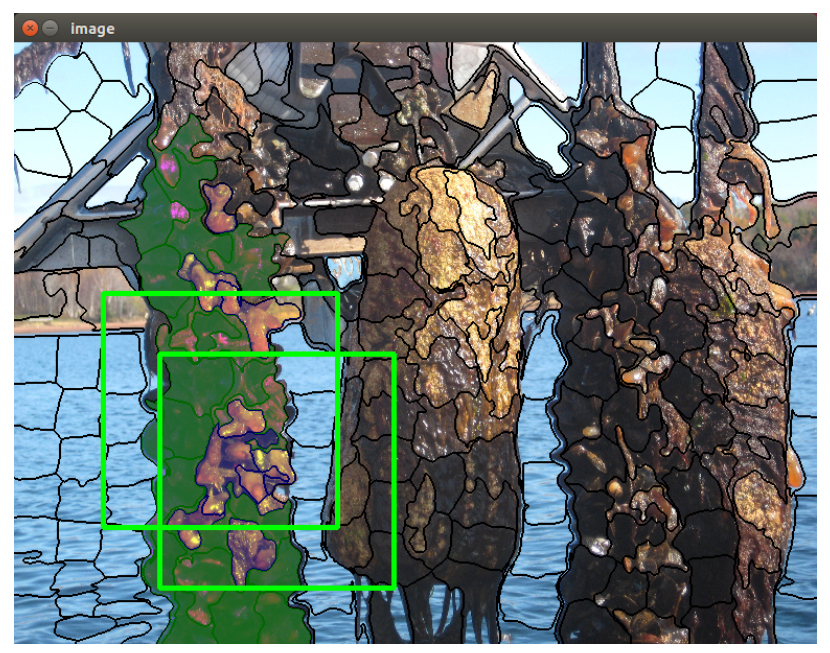

Figure 2. Screenshot of "Truth and Crop" utility with superpixel segments extracted via SLIC [15] for full size image downsampled by four (4), with $\sigma=3$, and 300 roughly equal sized segments. Two mussel socks, and two Styrofoam buoys are present, with lime-green bounding boxes where cropped regions are to be extracted. (Left) Ground truthed regions of sock have a transparent colour fill, with green for 1-Mussel, and purple for 2Ciona. Regions that are not filled are considered 0-Other after cropping operation. (Right) Image not ground truthed to show raw segments. Best viewed in colour.

made the labeling process faster. After labeling foreground classes, all other pixels were automatically assigned to the "Other" class.

Figure 3 depicts some of the challenging decisions that had to be made during the annotation process. Some difficult images resulted in superpixels that contained several classes, e.g. background and a mussel, or mussel and Ciona. In general, for cases where the annotator estimated that each class was roughly equally represented in a superpixel, the void label was assigned. Since all labels had to be assigned to a whole superpixel, the void label is more prominent than the thin border line that appears in PASCAL, however this was still considered efficient compared to manually drawing polygons with alternative tools such as LabelMe [16].

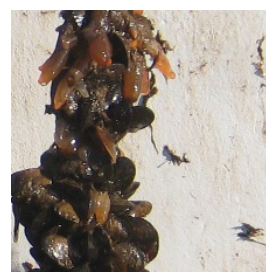

a)

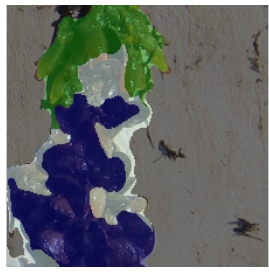

b)

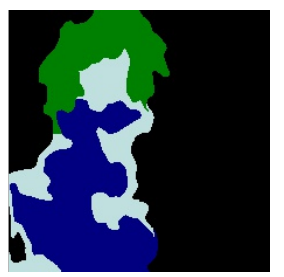

c)
Figure 3. Ciona17 sample of mussel sock top section from Farm 1. a) RGB image, $b$ ) segmentation mask imposed on $a$ ), and $c$ ) segmentation mask with Ciona as green area, mussels as blue area, and void as white area. Best viewed in colour.

The majority of superpixels resulted in much sharper edges in well illuminated areas than would have been 
feasible to draw in LabelMe. Superpixels that contained only Ciona and mussel were assigned the majority class. The void label was also used in some cases that were too difficult to discern, usually in areas with particularly low light.

To ensure good coverage of annotated regions, there was some overlap between images, similarly to what can be observed in Figure 2. We emphasize that for the splits outlined in Table [1 there was no overlap between the training and test sets as these images were cropped from different original images.

\section{Dataset Features}

We explained previously how the Ciona17 dataset relates to open problems in aquaculture. Now, a case is made for Ciona17 as a challenging and interesting computer vision dataset in general.

1) Variable Lighting: Although the weather was clear and sunny during both days that images were collected, sock sections cast in shadow make accurate segmentation difficult, even for the human eye. Figure 4 shows how challenging it can be to identify clusters of Ciona on mussel socks in broad daylight, due to a range of illumination and shadow inherent in outdoor imagery.

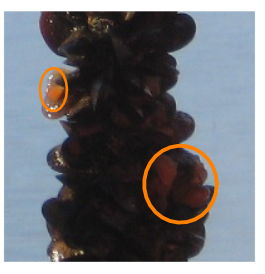

a)

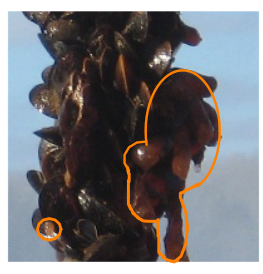

b)

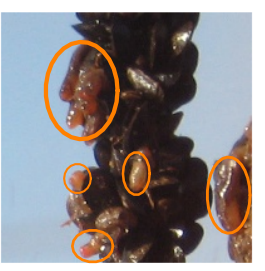

c)
Figure 4. Samples of $a$ ) "low", b) "medium", and c) "high" illumination sock sections taken from the same original image. Sections $a$ ) and $b$ ) are from the same sock, while $c$ ) is from an adjacent sock. All instances of Ciona were carefully annotated in orange. Best viewed in colour.

2) Colour: In theory, colour thresholding should separate mussels from the mostly bright orange Ciona, but this assumes direct sunlight, and lack of silt or debris. It has also been speculated that Ciona tend to darken with age [17]. The sample in Figure 5 shows a typical spectrum of colour for Ciona.

Additionally, some mussel shells reflect a similar orange colour to that of Ciona, an effect visible in Figure $4 b$ ) and $c$ ). This tends to occur naturally around the seam where the shell halves meet, but silt also contributes to a more global effect.

3) Texture: Styela clava are the mostly highly textured of all species in the dataset, but their colour is less distinguishable from mussels. Older Ciona have a finely pitted texture as their tunic thickens, while Styela consistently possess a coarse sandpaper-like appearance.

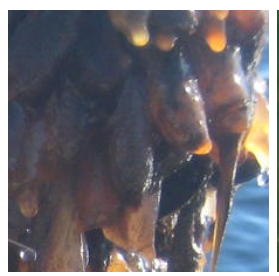

a)

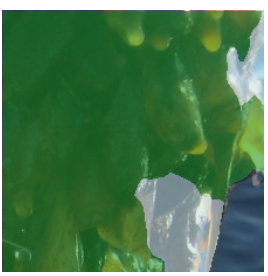

b)

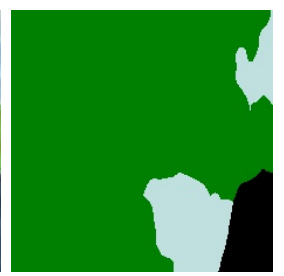

c)
Figure 5. Ciona17 sample of predominantly Ciona with diverse colour from Farm 2. a) RGB image, $b$ ) segmentation mask imposed on $a$ ), and $c$ ) segmentation mask with Ciona as green area, and void as white area. Two superpixels had to be voided due to mussel grouped together with debris and background. Best viewed in colour.

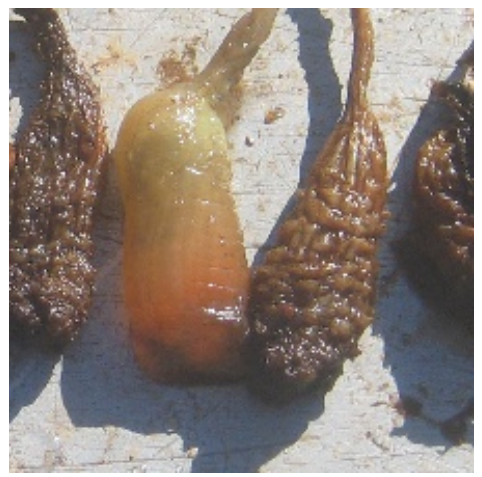

Figure 6. Sample of individual Ciona and Styela from Farm 2. Left Ciona and Right Styela. Best viewed in colour.

4) Occlusion: Farm 2 employed a technique known as "double socking" in which mussel socks are wrapped inplace with an additional mesh to prevent fall-off. This mesh can be considered as added occlusion in the dataset and is a common practice on some farms. The double socking made precise annotation extremely difficult, therefore we have held out this data for future consideration (e.g. for use in unsupervised or semi-supervised learning).

\section{Evaluating Performance}

To penalize naive schemes that optimize for background detection, we suggest using the standard "Mean Intersection over Union" (mIoU) metric, commonly used in semantic segmentation [18]. For a given class, and true positives (TP), false positives (FP), and false negatives (FN), the intersection over union (IoU) is given by:

$$
I o U=\frac{T P}{T P+F P+F N}
$$

Averaging the IoU scores for each class results in the overall mIoU score. In this way, a scheme that overwhelmingly predicts background will have little intersection with masks for mussels or Ciona, as no credit is earned for true negatives. 


\section{A. Baseline Result}

To establish a baseline mIoU score on the Ciona17 dataset, a variant of FCN [9] outlined in Table $[$ I], was trained end-to-end with pixelwise softmax cross-entropy loss and ReLU activations.

Table II

Fully CONVOlutional Network ARCHITECTURE (VGG6S-FC6-512-DECONV) USED AS OUR BASELINE.

\begin{tabular}{llcl}
\hline Layer Type & Kernel & Stride & Output Shape \\
\hline Input & n/a & n/a & $224 \times 224 \times 3$ \\
\hline Conv_1 & $3 \times 3$ & 1 & $224 \times 224 \times 16$ \\
Pool_1 & $2 \times 2$ & 2 & $112 \times 112 \times 16$ \\
\hline Conv_2 & $3 \times 3$ & 1 & $112 \times 112 \times 32$ \\
Pool_2 & $2 \times 2$ & 2 & $56 \times 56 \times 32$ \\
\hline Conv_3 & $3 \times 3$ & 1 & $56 \times 56 \times 64$ \\
Pool_3 & $2 \times 2$ & 2 & $28 \times 28 \times 64$ \\
\hline Conv_4 & $3 \times 3$ & 1 & $28 \times 28 \times 128$ \\
Pool_4 & $2 \times 2$ & 2 & $14 \times 14 \times 128$ \\
\hline Conv_5 & $3 \times 3$ & 1 & $14 \times 14 \times 256$ \\
Pool_5 & $2 \times 2$ & 2 & $7 \times 7 \times 256$ \\
\hline Fc_6 & $1 \times 1$ & 1 & $7 \times 7 \times 512$ \\
Deconv_1 & $64 \times 64$ & 32 & $224 \times 224 \times 3$ \\
\hline
\end{tabular}

Training was performed with the adaptive moment estimation (Adam) gradient descent scheme [19], initial learning rate of $1 \times 10^{-5}$, mini-batch size of 10 , and dropout with a dropout rate of 0.5 on layer $\mathrm{FC}_{-} 6$. The RGB images were not preprocessed other than a simple conversion of the 8bit pixel intensities to floating point values in the range [01]. Training was interrupted at $100 \mathrm{k}$ steps, re-starting the optimizer from scratch, and allowed to continue until 300k total steps had elapsed resulting in $m I o U_{\text {test }}=51.36 \%$. Some samples that were drawn from the trained model are shown in Figure 7

In practice, it may be acceptable to merge the "other" and "mussel" classes, turning the problem into that of foreground/background binary segmentation, as a closed loop treatment system only needs to distinguish Ciona from everything else. Despite this, farmers may wish to contextualize the Ciona measure with mussel biomass, hence the need to report IoU across all classes.

\section{CONCLUSiON}

To the best of the authors' knowledge, Ciona17 is the first dataset of its kind with pixel-level annotations pertaining to invasive species in a marine environment. It is the authors' intent that in making this dataset available, the research community will propose new models that are particularly adept at controlling for highly variable illumination and occlusions, exceeding the initial benchmark mIoU of $51.36 \%$.

In making the original images and tools used for annotation available, we expect that improved ways of annotating challenging datasets such as this one will be proposed.
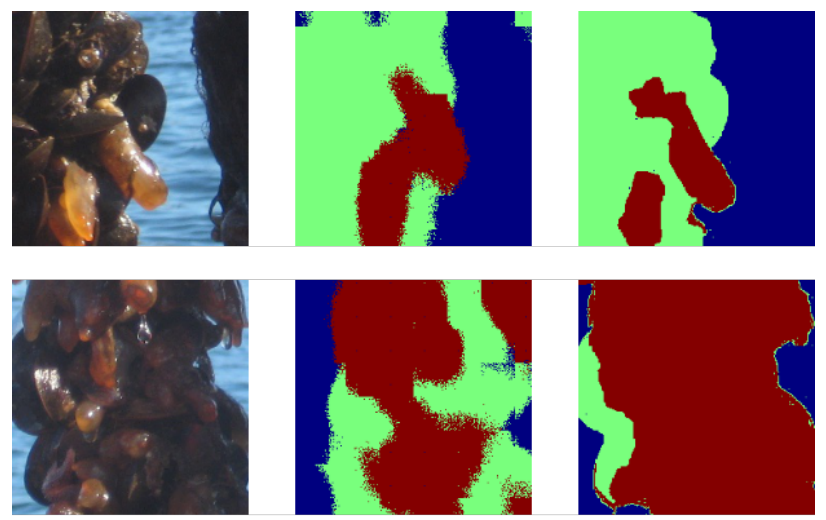

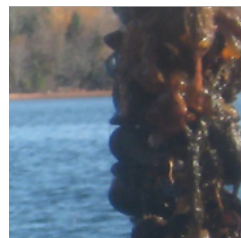

a)

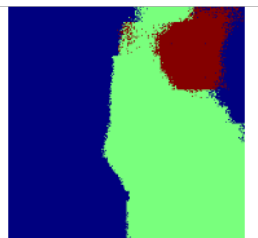

b)

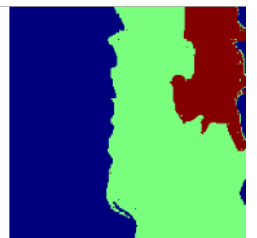

c)
Figure 7. Three sets of $a$ ) RGB image sample from Ciona17 test set, $b$ ) prediction of trained VGG6S model, and c) segmentation mask with Ciona as red, mussels as green, and other as blue. Best viewed in colour.

\section{ACKNOWLEDGMENT}

The authors thank Brian Gillis of the PEI Department of Agriculture and Fisheries for assisting with the data acquisition. We also thank Colin Reynolds of Reynold's Island Mussels, and Dana Drummond's crew at Atlantic Aqua Farms for offering their farms for data collection.

\section{REFERENCES}

[1] A. Ramsay, J. Davidson, D. Bourque, and H. Stryhn, "Recruitment patterns and population development of the invasive ascidian ciona intestinalis in Prince Edward Island, Canada," Aquatic Invasions, vol. 4, 2009.

[2] C. Paetzold, D. Giberson, J. Hill, and J. Davidson, "Effect of colonial tunicate presence on ciona intestinalis recruitment within a mussel farming environment," Management of Biological Invasions, vol. 3, 2012.

[3] J. Davidson, T. Landry, G. Johnson, and P. Quijón, "A costbenefit analysis of four treatment regimes for the invasive tunicate ciona intestinalis on mussel farms," Management of Biological Invasions, vol. 8, 2016.

[4] A. Gittenberger, "Invasive tunicates on Zeeland and Prince Edward Island mussels, and management practices in The Netherlands," Aquatic Invasions, vol. 4, 2009.

[5] G. M. Moeser and E. Carrington, "Seasonal variation in mussel byssal thread mechanics," The Journal of Experimental Biology, 2006.

[6] M. J. O’Donnell, M. N. George, and E. Carrington, "Mussel byssus attachment weakened by ocean acidification," Nature Climate Change, vol. 3, pp. 587-590, 2013. 
[7] Prince Edward Island Aquaculture Alliance, "Final report: The development and early commercialization of four innovative tunicate treatment systems for mussel aquaculture on PEI," tech. rep., Department of Fisheries and Oceans Aquaculture and Innovation Market Access Program, March 2010.

[8] M. Everingham, L. Van Gool, C. K. I. Williams, J. Winn, and A. Zisserman, "The PASCAL Visual Object Classes Challenge 2012 (VOC2012) Results." http://www.pascalnetwork.org/challenges/VOC/voc2012/workshop/index.html.

[9] J. Long, E. Shelhamer, and T. Darrell, "Fully convolutional networks for semantic segmentation," in CVPR, 2015.

[10] H. Noh, S. Hong, and B. Han, "Learning deconvolution network for semantic segmentation," in ICCV, vol. 00, pp. 1520$1528,2015$.

[11] L.-C. Chen, G. Papandreou, I. Kokkinos, K. Murphy, and A. L. Yuille, "Semantic image segmentation with deep convolutional nets and fully connected crfs," in ICLR, 2015.

[12] M. Cordts, M. Omran, S. Ramos, T. Rehfeld, M. Enzweiler, R. Benenson, U. Franke, S. Roth, and B. Schiele, "The cityscapes dataset for semantic urban scene understanding," in Proc. of the IEEE Conference on Computer Vision and Pattern Recognition (CVPR), 2016.

[13] M. Abadi, A. Agarwal, P. Barham, E. Brevdo, Z. Chen, C. Citro, G. S. Corrado, A. Davis, J. Dean, M. Devin, S. Ghemawat, I. Goodfellow, A. Harp, G. Irving, M. Isard, Y. Jia, R. Jozefowicz, L. Kaiser, M. Kudlur, J. Levenberg, D. Mané, R. Monga, S. Moore, D. Murray, C. Olah, M. Schuster, J. Shlens, B. Steiner, I. Sutskever, K. Talwar, P. Tucker, V. Vanhoucke, V. Vasudevan, F. Viégas, O. Vinyals, P. Warden, M. Wattenberg, M. Wicke, Y. Yu, and X. Zheng, "TensorFlow: Large-scale machine learning on heterogeneous systems," 2015. Software available from tensorflow.org.

[14] R. Achanta, A. Shaji, K. Smith, A. Lucchi, P. Fua, and S. Susstrunk, "Slic superpixels compared to state-of-the-art superpixel methods," IEEE Trans. Pattern Anal. Mach. Intell., vol. 34, pp. 2274-2282, Nov. 2012.

[15] F. Pedregosa, G. Varoquaux, A. Gramfort, V. Michel, B. Thirion, O. Grisel, M. Blondel, P. Prettenhofer, R. Weiss, V. Dubourg, J. Vanderplas, A. Passos, D. Cournapeau, M. Brucher, M. Perrot, and E. Duchesnay, "Scikit-learn: Machine learning in Python," Journal of Machine Learning Research, vol. 12, pp. 2825-2830, 2011.

[16] B. C. Russell, A. Torralba, K. P. Murphy, and W. T. Freeman, "LabelMe: A database and web-based tool for image annotation," 2008.

[17] A. Ramsay. Private Communication, 2017. PEI Department of Agriculture and Fisheries.

[18] M. Everingham, S. M. A. Eslami, L. Van Gool, C. K. I. Williams, J. Winn, and A. Zisserman, "The Pascal visual object classes challenge: A retrospective," International Journal of Computer Vision, vol. 111, no. 1, pp. 98-136, 2015.

[19] D. P. Kingma and J. Ba, "Adam: A method for stochastic optimization," in ICLR, 2015. 